\title{
APPLICATION OF COMPUTATIONAL INTELLIGENCE TO TARGET TRACKING
}

\author{
Lars Nolle \\ Computational Optimisation and Applications Research Group \\ School of Computing and Informatics \\ Nottingham Trent University \\ Clifton Lane, Nottingham, NG11 8NS, UK \\ lars.nolle@ntu.ac.uk
}

\section{KEYWORDS}

Target Motion Analysis, Robust Estimators, Ant Colony Optimisation.

\begin{abstract}
In the oceanic context, the aim of Target Motion Analysis (TMA) is to estimate the state, i.e. location, bearing and velocity, of a sound-emitting object. These estimates are based on a series of passive measures of both the angle and the distance between an observer and the source of sound, which is called the target. These measurements are corrupted by noise and false readings, which are perceived as outliers.
\end{abstract}

Usually, sequences of measurements are taken and statistical methods, like the Least Squares method or the Annealing M-Estimator, are applied to estimate the target's state by minimising the residual in range and bearing for a series of measurements.

In this project, an ACO-Estimator, a novel hybrid optimisation algorithm based on Computational Intelligence, has been developed and applied to the TMA problem and its effectiveness was compared with standard estimators. It was shown that the new algorithm outperforms conventional estimators by successfully removing outliers from the measurements.

\section{INTRODUCTION}

The aim of Target Motion Analysis (TMA) in the maritime context is to predict the state, i.e. location, bearing and velocity, of a signal-emitting object, also known as the target, based on previous observations (Hassab et al., 1981).

The observer receives signals that are emitted from the target where the time of emission is not known. The range $\mathrm{R}$ and the bearing of the target are usually determined by measuring differences in arrival time of short-duration acoustic emissions along the paths $R_{l}, R$ and $R_{2}$ of a target $T$ using hydrophones that are mounted at some distance $D$ on a cable towed by an observer platform (Figure 1).

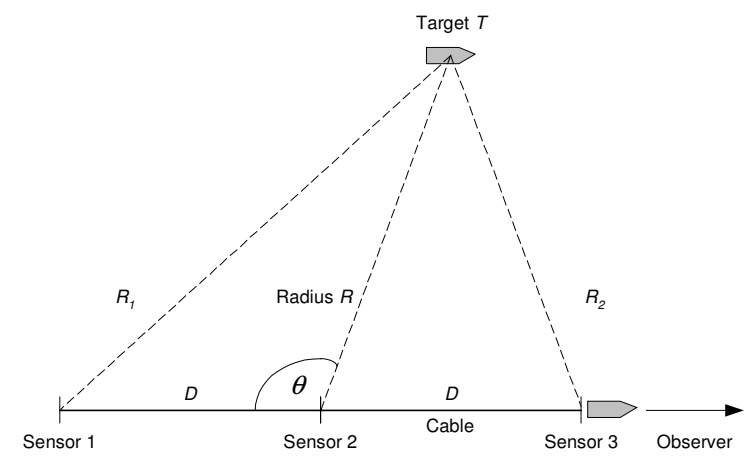

Figure 1 - Typical scenario for the Target Motion Analysis problem.

In a real application, the time delay measurements are disturbed by noise, caused, for example, by the crosscorrelation function used for finding a common signal in a pair of sensors or by the environment (Carevic, 2003). The time delay error distribution function is a non-Gaussian one. Another source of errors is false readings or clutter. This clutter is usually assumed to be uniformly distributed over an area $A$ and to follow a Poisson probability density function. Figure 2 shows a typical scenario for the TMA problem. An observer and a target are moving with constant speed and the target is detected at unequally spaced time instances by the observer.

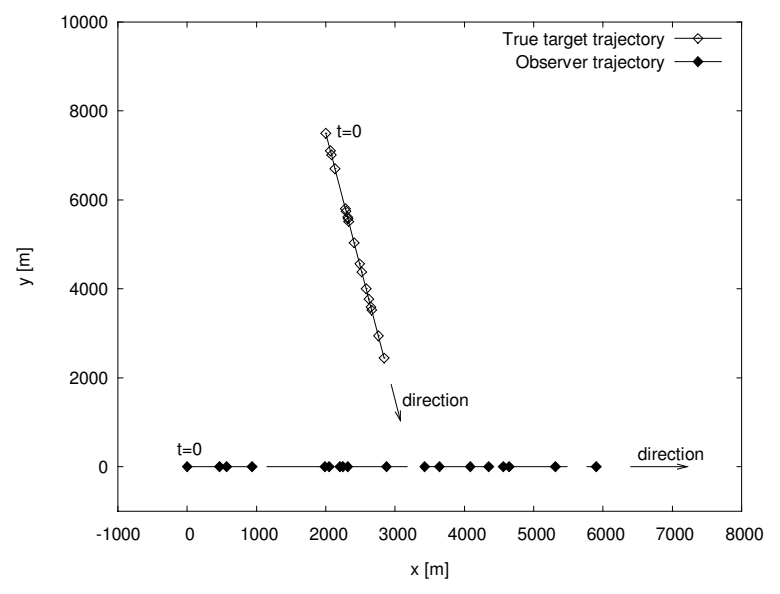

Figure 2 - Typical scenario for the Target Motion Analysis problem. 
Figure 3 shows the noisy measurements and clutter for the same scenario. Both types of errors introduce additional complexity to the target state estimation problem.

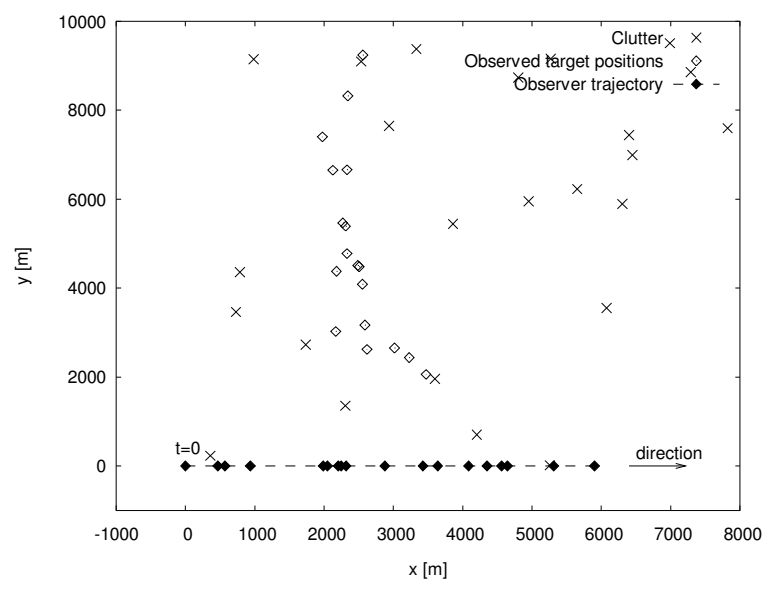

Figure 3 - Noisy measurements and clutter for scenario given in Figure 2.

Usually, the measurements are taken and statistical methods are applied to estimate the targets' state. In recent research, it was shown that for the TMA problem with range and bearing measurements an Annealing M-Estimator ( $\mathrm{Li}, 1996)$ outperforms traditional methods in highly cluttered environments (Carevic, 2003). This type of estimator reduces the influence of outliers by applying a weighting function to the measurements. Obviously, better results could be achieved if the clutter, i.e. the outliers, were not used at all in the estimation process.

The aim of this research was to develop an intelligent estimator that has the ability to decide whether or not a particular measurement is clutter. The new estimator developed is referred to as ACO-Estimator.

\section{ACO-ESTIMATOR}

The ACO-Estimator is based on Ant Colony Optimisation (ACO) (Dorigo and De Caro, 1999). ACO refers to a class of discrete optimisation algorithms, i.e. a Computational Intelligence (CI) meta-heuristic, which is modelled on the collective behaviour of ant colonies.

The main principle of ACO is that a colony of artificial ants builds probability distributions for each input parameter of a system to be optimised. Initially, every possible choice for each of the input variables is set to a very low probability, which is the equivalent of the pheromone level in the real world (Dorigo and De Caro, 1999). Each individual ant then chooses one value for each input parameter, i.e. builds up a candidate solution, called a trail vector, based on the probability distributions of the input values. Depending on the quality of the resulting candidate solution, the probability values of the chosen input values are updated. The whole process is repeated in iterations called time steps until a suitable solution is found or the algorithm has converged, i.e. has reached a stable set of probability distributions.

For the ACO-Estimator, each measurement in a set is an input, which can either have the state 'included' or 'excluded', i.e. 'on' or 'off'. Artificial ants 'travel' through the state space, choosing their paths based on the associated pheromone levels. Based on the achieved fitness, which is related to the mean residuals achieved using local search and the Least Squares (LS) method, the pheromone levels, i.e. probabilities, are adjusted. Figure 4 shows the flowchart of the ACOEstimator.

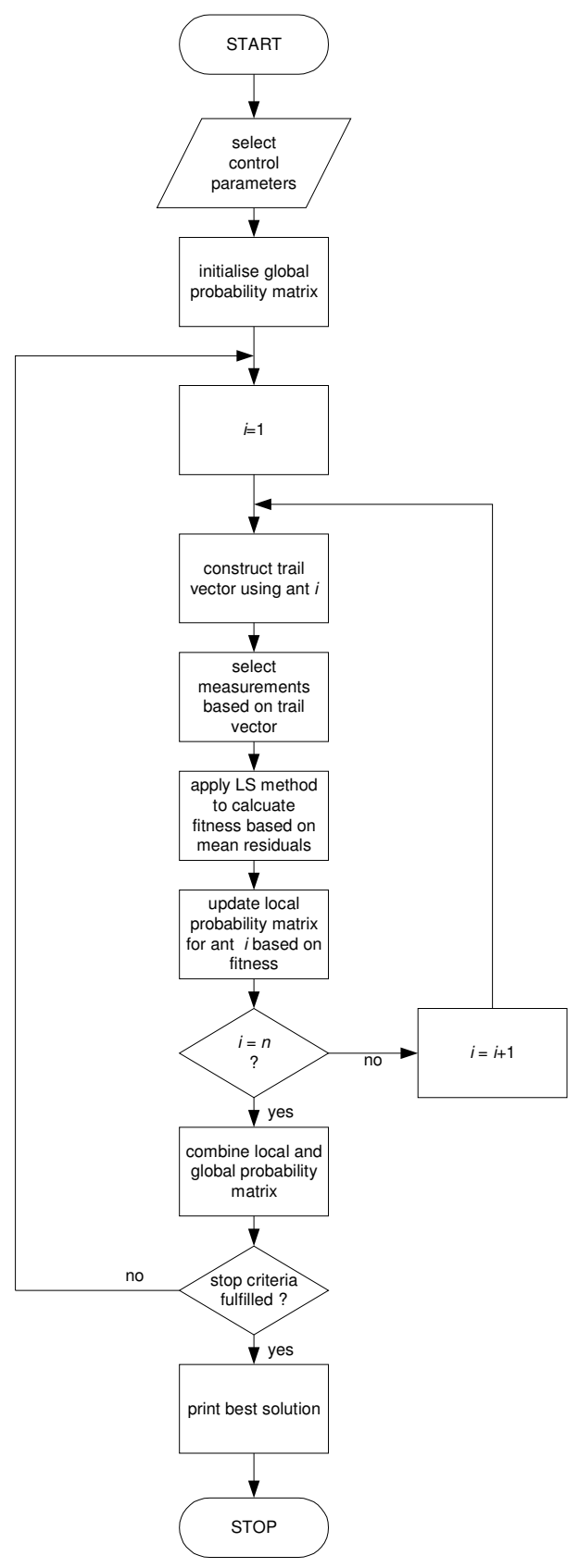

Figure 4 - Flowchart of the ACO-Estimator. 


\section{EXPERIMENTS}

Two of the four tracking scenarios proposed by Carevic (2003) have been used in this work: scenario 1 and scenario 4.

For both scenarios, datasets with seven different mean percentages of clutter (MPC) have been generated. The MPC was varied from $0 \%$ to $60 \%$ in order to cover the whole range up to $50 \%$, which is the theoretical breakdown point for robust estimators (Rousseeuw and Leroy, 1987). For each MPC, 100 sets were generated resulting in a total of 1,400 data sets, i.e. experiments. The Mean Trajectory Distances (MTDs) were used as the fitness function. Because the TMA problem is a semi real-time application, the maximum number of fitness evaluations was limited to 200,000 for each of the experiments

The new estimator was compared with three standard estimators, the LS estimator, the Iteratively Reweighted Least Squares estimator (IRLS) (Hong and Chen, 2005), and the Annealing MEstimator (AM) (Li, 1996).

For each of the 100 simulations in an MPC sets, the average mean trajectory distance (MMTD) for that group and their standard deviations (STD) were calculated.

Figure 5 shows a graphical representation of the results for the MMTDs obtained from the experiments for tracking scenario 1, Figure 6 shows the MMTDs for tracking scenario 4. Figure 7 and Figure 8 present graphical representations of the achieved STDs of the MMTDs.

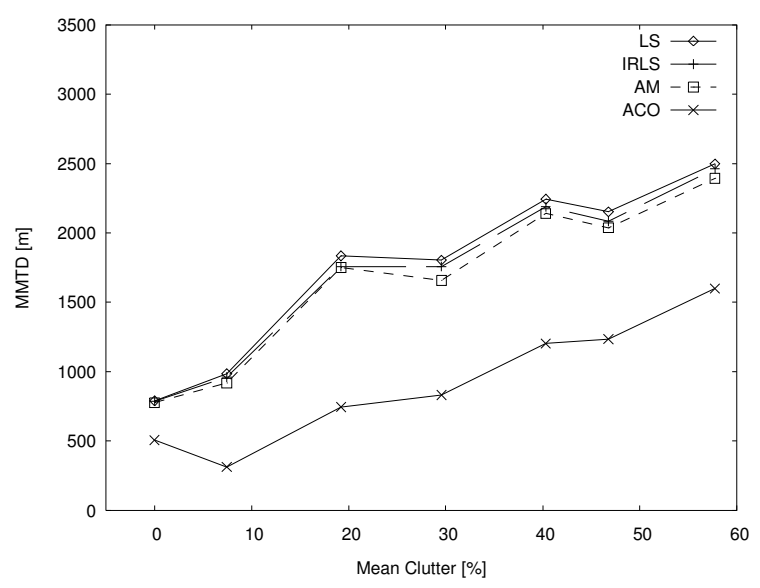

Figure 5 - Average Mean Trajectory Distance (MMTD) for scenario 1.

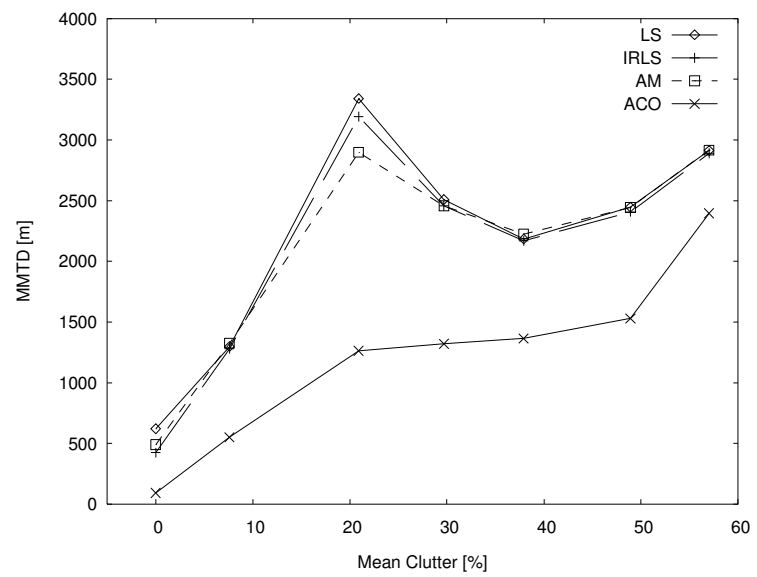

Figure 6 - Average Mean Trajectory Distance (MMTD) for scenario 4.

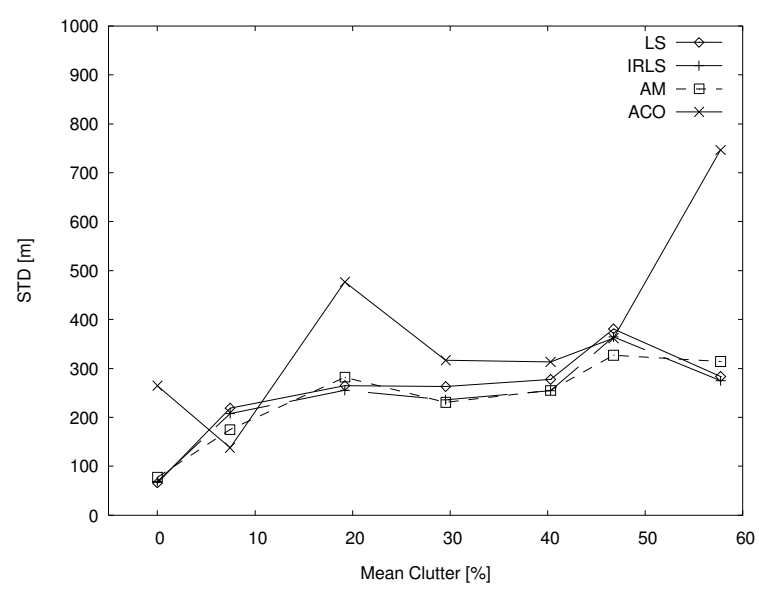

Figure 7 - Standard deviation of average Mean Trajectory Distance (MMTD) for scenario 1.

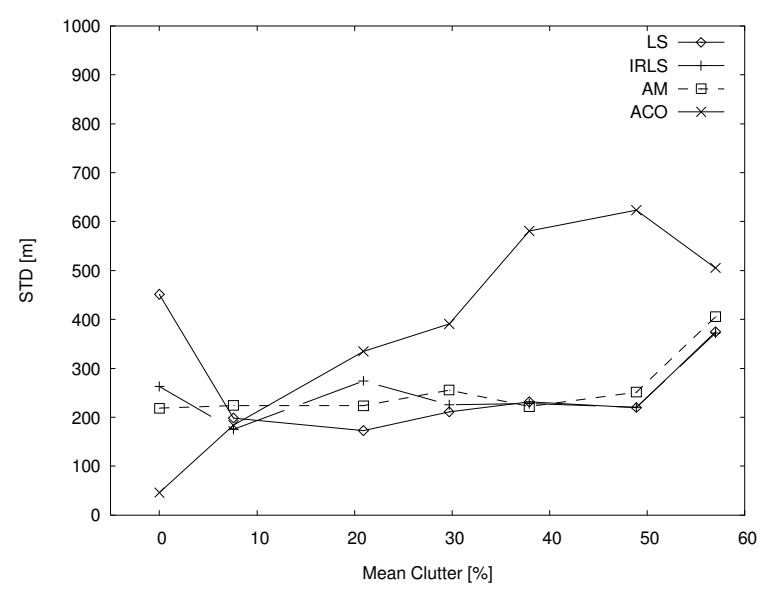

Figure 8 - Standard deviation of average Mean Trajectory Distance (MMTD) for scenario 4. 


\section{DISCUSSION}

As it can be seen from Figure 5 and Figure 6, for both tracking scenarios the three conventional estimators showed very similar performances, although the AM-Estimator performed slightly better. This is in agreement with the findings reported by Carevic (2003).

Table 1 shows the average improvements in terms of MMTD achieved using the ACO-Estimator. It can be seen that the overall improvement is $46.3 \%$ for scenario 1 and $43.1 \%$ for scenario 4 . Therefore, the new ACO-Estimator outperformed all of the other estimators used in terms of MMTD.

\begin{tabular}{|c|c|c|}
\hline \multirow{2}{*}{} & \multicolumn{2}{|c|}{$\begin{array}{r}\text { Average Improvement of MMTD } \\
\text { for ACO-Estimator }\end{array}$} \\
\cline { 2 - 3 } & Scenario 1 [\%] & Scenario 4 [\%] \\
\hline LS & 47.7 & 44.4 \\
\hline IRLS & 46.4 & 42.6 \\
\hline AM & 44.9 & 42.3 \\
\hline
\end{tabular}

Table 1 - Average improvement of MMTD.

However, in terms of STD, the performance of the ACO-Estimator was worse for both tracking scenarios (Table 2).

\begin{tabular}{|c|c|c|}
\hline \multirow{2}{*}{} & \multicolumn{2}{|c|}{$\begin{array}{c}\text { Average Improvement of STD for } \\
\text { ACO-Estimator }\end{array}$} \\
\cline { 2 - 3 } & Scenario 1 [\%] & Scenario 4 [\%] \\
\hline \multirow{2}{*}{ LS } & -48.9 & -43.3 \\
\hline IRLS & -57.6 & -51.5 \\
\hline AM & -57.6 & -48.0 \\
\hline
\end{tabular}

Table 2 - Average improvement of STD.

The ACO-Estimator had problems with full converge within the time available for the estimations, especially when the amount of MPC was high (Figure 7 and Figure 8). Figure 9 shows a typical run of the algorithm without time limitations.

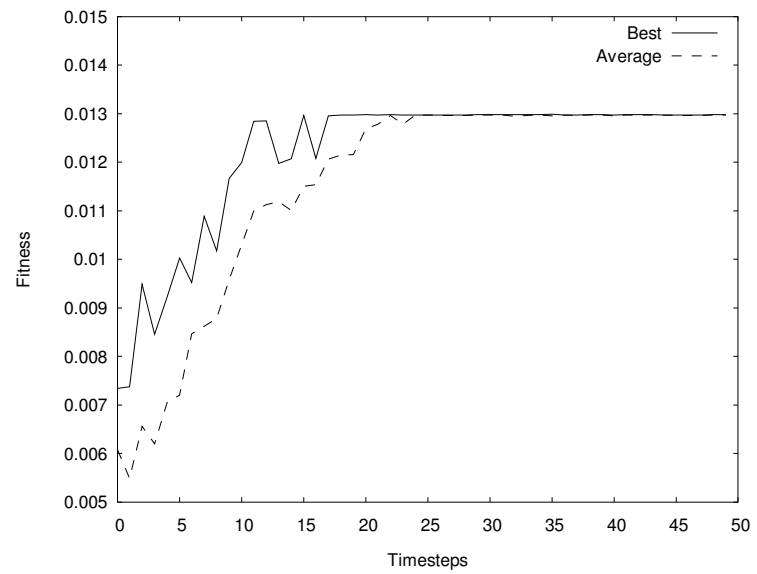

Figure 9 - Typical search run without time limitations.

As it can be seen, the algorithm converges after approximately 24 time steps, whereas the algorithm usually carried out 11 time steps in the experiments before the time available elapsed.

\section{CONCLUSION}

The main aim of this research project was to develop a new robust estimation method that has the ability to solve the linear array-based TMA problem. This has been achieved by developing a new ACO-based estimation algorithm, which was implemented and successfully applied to the TMA problem. Based on the statistical analysis of the results obtained from the experiments, it was concluded that the new algorithm outperforms conventional estimators by successfully removing outliers from the measurements.

\section{AUTHOR BIOGRAPHY}

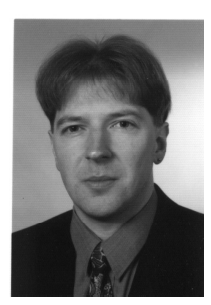

Lars Nolle graduated from the University of Applied Science and Arts in Hanover in 1995 with a degree in Computer Science and Electronics. After receiving his $\mathrm{PhD}$ in Applied Computational Intelligence from The Open University, he worked as a System Engineer for EDS. He returned to The Open University as a Research Fellow in 2000. He joined the Nottingham Trent University as a Senior Lecturer in Computing in February 2002. His research interests include: applied computational intelligence, distributed systems, expert systems, optimisation and control of technical processes. 


\section{REFERENCES}

Carevic, D. (2003) 'Robust Estimation Techniques for Target Motion Analysis Using Passively Sensed Transient

Signals', IEEE Journal of Oceanic Engineering, Vol. 28, No. 2, April, pp 262-270.

Dorigo, M., De Caro, G. (1999) 'The Ant Colony Optimization Meta-Heuristic', in: Corne, D., Dorigo, M., Glover, F. (Eds) New Ideas in Optimization, McGrawHill, pp. 11-32.

Hassab, J. C., Guimond, B.W., Nardone, S. C. (1981) 'Estimation of location and motion parameters of moving source observed from a linear array', Journal of the Acoustical Society of America, Vol. 70, No. 4, October, pp 1054-1061.

Hong, X., Chen, S. (2005) 'M-Estimator and D-Optimality Model Construction Using Orthogonal Forward Regression', IEEE Transactions on Systems, Man, And Cybernetics - Part B, Vol. 5, No 1, pp155-162.

Li, S.Z. (1996) 'Robustizing Robust M-Estimation Using Deterministic Annealing', Pattern Recognition, Vol. 29, No. 1, pp 159-166.

Rousseeuw, P.J., Leroy, A.M. (1987) Robust Regression and Outlier Detection, John Wiley, New York. 